\title{
Social Support in Normal Pressure Hydrocephalus: Unmet Tangible Social Support
}

\author{
Alexander McGirr, Michael D. Cusimano
}

Can J Neurol Sci. 2013; 40: 94-96

Idiopathic Normal Pressure Hydrocephalus (iNPH) is a syndrome described as a triad of gait unsteadiness, urinary incontinence and memory impairment in the context of ventriculomegaly and normal cerebrospinal fluid (CSF) pressure $^{1}$. Idiopathic Normal Pressure Hydrocephalus is a potentially heritable condition ${ }^{2}$, for which surgical intervention is available that, while of unproven efficacy, results in improvement in a significant proportion of patients and a partial return to function.

Chronic illness results in a significant burden upon the individual and their caregivers. Social support has been found to result in improved quality of life in addition to improving survival in a variety of conditions. Like other chronic conditions, iNPH imposes a significant burden on caregivers, with preliminary evidence that CSF diversion surgery results in a decreased burden on caregivers ${ }^{3}$.

To our knowledge, adult hydrocephalus, whether of congenital, acquired or idiopathic origin, has not been characterized with respect to social support and its role in function or long-term outcome. It is unfortunate that despite clear modes of intervention through support groups and social services, the social support needs of patients with iNPH have not been described.

However, in the paediatric hydrocephalus literature, there is data to suggest that measures with face resemblance to social support are associated with improved outcomes ${ }^{4}$. In addition to common sociodemograhic indicators, an association has been reported between caregiver's report of family functioning and children with hydrocephalus's cognitive, physical, socioemotional and overall health ${ }^{4}$. While it is unclear whether poorer family functioning leads to poorer outcome or is consequent to a child's outcome, it is clear that family functioning and social support are deserving of further investigation in conditions characterized by hydrocephalus.

In this study, we aimed to characterize the perceived social support of patients with iNPH. In so doing, we aimed to identify unmet needs and areas for potentially effective intervention that might improve this population's function and quality of life. While disease process matched controls would provide an evaluation of iNPH-specific needs, in the absence of data suggesting social support lacunae in other forms of hydrocephalus, we first aimed to characterize whether this population has unmet social support needs. As shunting may improve function and decrease social support needs, we chose to study patients having already undergone ventriculoperitoneal CSF diversion.

\section{Methods}

This characterization took place alongside a family study of iNPH described elsewhere ${ }^{2}$. Research Ethics Board approval was obtained at St-Michael's Hospital and participants provided written informed consent.

\section{iNPH patients}

We identified 690 patients having undergone ventriculoperitoneal CSF diversion at St-Michael's Hospital from 20042010. From this list, 52 were identified with a pre-operative diagnosis of iNPH.

An invitation letter was sent and followed-up by telephone. Of the initial cases, fifteen cases could not be recruited due to death $(n=10)$ or invalid contact information $(n=5)$. Of the remainder, $21(56.7 \%)$ patients returned the study questionnaire package.

We have previously shown moderate to excellent reliability and validity of iNPH self-report using information obtained from proxy-patient pairs $^{2}$.

\section{Control Subjects}

A priori, we identified control probands using the acquaintanceship method where cases are asked to name a family friend (non-relative) of similar age and sex to serve as a control. This method minimizes differences in sex, age, ethnicity, marital status, socioeconomic status, education and family density. Ten NPH cases identified controls using this approach. Eleven control participants were identified among neurosurgical patients seen at St-Michael's Hospital (acoustic neuroma $n=1$, glioma $n=1$, cervical fracture $n=2$, lumbar spondylolisthesis $n=4$, lumbar stenosis $n=1$, spinal metastases $\mathrm{n}=2$ ).

\section{Assessment}

Participants completed the MOS Social Support Survey questionnaire $^{5}$, a validated 18 -item instrument assessing perceived social support, specifically emotional/informational

\footnotetext{
From the Division of Neurosurgery, St-Michael's Hospital, University of Toronto, Toronto, Ontario, Canada.

Received April 3, 2012. Final Revisions Submitted July 27, 2012. Correspondence to: Alexander McGirr, Injury Prevention Research Office, St. Michael's Hospital, 30 Bond Street, Toronto, Ontario, M5B 1W8, Canada. Email: alexander.mcgirr@alum.utoronto.ca.
} 


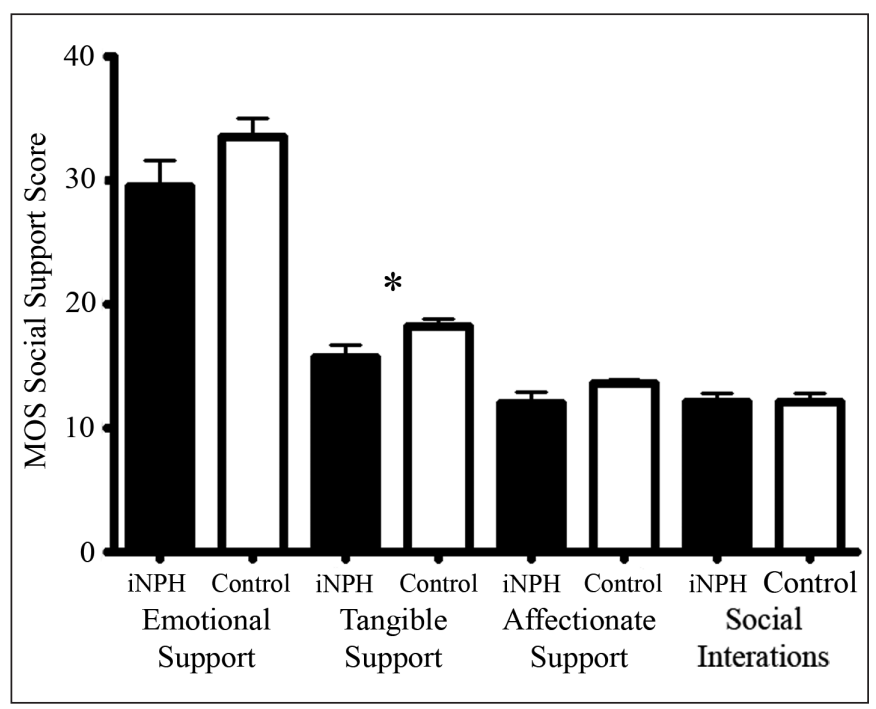

Figure: Perceived Positive Social Support in Idiopathic Normal Pressure Hydrocephalus

support (eight items), tangible support (four items), affectionate support (three items), and positive social interaction (three items). Items are rated on a 5 point Likert scale $(1=$ 'None of the time', $3=$ 'Some of the time', $5=$ 'All of the time'). Thus, higher scores represent a greater degree of social support. Internal consistency for the current sample was excellent on all domains (tangible $\alpha=.95$, affection $\alpha=.90$, social $\alpha=.95$, and emotional $\alpha=.94)$.

\section{Statistical Analyses}

We performed analyses using the SPSS statistical package version 19 (SPSS Inc., Chicago, IL). Data distributions were checked for normality. Chi square tests were used for categorical measures, student t-test was used for continuous variables. Significance was set at $\alpha<0.05$.

\section{RESUlts}

Demographic characteristics for this sample are presented in the Table and elsewhere ${ }^{2}$. This primarily Caucasian sample was matched for age $(73.29 \pm 7.66$ vs $71.24 \pm 7.55)$, sex $(38.1 \%$ vs $42.9 \%$ male), marital status $(61.9 \%$ vs $61.9 \%$ married), education (61.9\% vs $57.1 \%$ technical degree or higher), household income $(14.3 \%$ vs $14.3 \%$ household income $>\$ 100,000)$, proportion living alone $(38.1 \%$ vs $33.3 \%)$, and family density ${ }^{2}$.

Results with respect to MOS social support domains are presented in Figure 1. iNPH patients rated their tangible support significantly lower than did control patients $(15.71 \pm 4.74 \mathrm{vs} 18.19 \pm 2.85, \mathrm{t}(40)=2.04, \mathrm{p} \leq .05)$. No differences were noted with respect to emotional support $(29.52 \pm 9.67$ vs33.47 $\pm 7.27, \mathrm{t}(40)=1.49, \mathrm{p}=.142)$, affectionate support $(12.00 \pm 4.07 \mathrm{vs} 13.57 \pm 1.80, \mathrm{t}(40)=1.61, \mathrm{p}=.114)$ or positive social interactions $(12.09 \pm 3.30 \mathrm{vs} 12.09 \pm 3.31, \mathrm{t}(40)=.00, \mathrm{p}=1.00)$.

\section{Discussion}

To our knowledge, iNPH patients' perceived social support has not been characterized. In this study, patients with iNPH having undergone surgical treatment reported lower levels of tangible social support than age-, sex-, education- and family density-matched comparison subjects.

As tangible social supports relate to supports directed at instrumental activities of daily living, such as ambulation, transportation, meal preparation and cleaning, our data reflects, in part, the important physical limitations experienced by this population despite treatment. Yet, large population studies using the MOS social support survey have found no relationship between physical functioning and perceived tangible social support, and only weak associations with overall assessments of physical health 5 . Instead, the tangible support domain appears to serve as a proxy for social isolation, limited social activities, as well as dysfunctional family and marital dynamics ${ }^{5}$. Moreover, the tangible support domain has been associated with lower emotional quality of life and subsequent development of clinically significant depression.

Our data, therefore, suggests that lower levels of tangible support in the absence of other social support deficits may reflect strained familial supports in the face of the important physical and emotional burden of iNPH. This has clear implications with respect to the patient, but also the high potential for caregiver exhaustion as has been reported in neurodegenerative diseases with overlapping symptomatology. More important still, it highlights the importance of developing and maintaining social support groups and social services in this population.

Our approach to comparison subject selection achieved an excellent degree of matching, and therefore differences are more likely to represent iNPH specific processes and not established relationships with respect to sociodemographic factors. Our findings are, nevertheless, limited by a small sample size and a single measure of social support. Our sample size limits statistical power $(71.5 \%$ power for two tailed t-tests), and

Table: Demographic characteristics

\begin{tabular}{l|c|c|c}
\hline & $\begin{array}{c}\text { iNPH } \\
\% \text { or } \mathrm{M} \pm \mathrm{SD}\end{array}$ & $\begin{array}{c}\text { Comparison } \\
\% \text { or } \mathrm{M} \pm \mathrm{SD}\end{array}$ & $p$ \\
\hline Age & $73.29 \pm 7.66$ & $71.24 \pm 7.55$ & .23 \\
\hline Sex & $38.1 \%$ & & .75 \\
\hline Male & $61.9 \%$ & $57.1 \%$ & 1.00 \\
\hline Female & $61.9 \%$ & $61.9 \%$ & .75 \\
\hline Married & $61.9 \%$ & $57.1 \%$ & 1.00 \\
\hline $\begin{array}{l}\text { Technical Degree } \\
\text { or Higher }\end{array}$ & $14.3 \%$ & $14.3 \%$ & .74 \\
\hline $\begin{array}{l}\text { Income } \\
>\$ 100,000 / \text { year }\end{array}$ & $38.1 \%$ & $33.3 \%$ & 1.00 \\
\hline Lives Alone & $90.5 \%$ & $90.5 \%$ & .29 \\
\hline Has Children & $2.53 \pm 1.21$ & $2.16 \pm .89$ & .72 \\
\hline $\begin{array}{l}\text { Biological } \\
\text { Children }\end{array}$ & $.16 \pm .37$ & $.21 \pm .53$ & \\
\hline $\begin{array}{l}\text { Non-Biological } \\
\text { Children }\end{array}$ & & & \\
\hline
\end{tabular}


therefore caution is required when interpreting non-significant comparisons for other sub-domains, such as emotional support. Moreover, our comparison subjects were not disease matched controls and iNPH symptom severity was not reassessed prior to completing the instrument and therefore replication will be required to ensure generalizability.

The role of social support groups and their potential benefit in this population should be further assessed. Future studies should involve a larger sample, prospectively measure the relationship between social support, quality of life and functional outcome, as well as involve disease process matched controls. Moreover, the role of CSF diversion in improving required supports should be assessed using a prospective design.

\section{CONCLUSION}

Our data suggests that patients with iNPH require additional social services and more aids than are currently allocated. Strained tangible social supports may reflect the physical and emotional challenges facing this population, even after surgical intervention, and likely reflect the significant burden borne by family members ${ }^{3}$.

\section{REFERENCES}

1. Adams RD, Fisher CM, Hakim S, Ojemann RG, Sweet WH. Symptomatic Occult Hydrocephalus with "Normal" Cerebrospinal-Fluid Pressure. A Treatable Syndrome. N Engl J Med. 1965 Jul 15;273:117-26.

2. McGirr A, Cusimano MD. Familial Aggregation of Idiopathic Normal Pressure Hydrocephalus: novel familial case and a family study of the NPH triad in an iNPH patient cohort. J Neurol Sci. 2012 Oct 15;321(1-2):82-8. Epub 2012 Aug 23.

3. Kazui H, Mori E, Hashimoto M, Ishikawa M, Hirono N, Takeda M. Effect of shunt operation on idiopathic normal pressure hydrocephalus patients in reducing caregiver burden: evidence from SINPHONI. Dement Geriatr Cogn Disord. 2011;31(5): 363-70.

4. Kulkarni AV, Cochrane DD, McNeely PD, Shams I. Medical, social, and economic factors associated with health-related quality of life in Canadian children with hydrocephalus. J Pediatr. 2008 Nov;153(5):689-95.

5. Sherbourne CD, Stewart AL. The MOS Social Support Survey. Soc Sci Med. 1991;32(6):705-14. 